\title{
HUNTING FOR YOUNG DISPERSING STAR CLUSTERS IN IC 2574
}

\author{
Anne Pellerin ${ }^{1,2,6}$, Martin M. Meyer $^{3}$, Daniella Calzetti ${ }^{4}$, and Jason Harris ${ }^{5}$ \\ ${ }^{1}$ Department of Physics and Astronomy, Texas A\&M University, College Station, TX 77843-4242, USA; apellerin@mta.ca \\ ${ }^{2}$ George P. and Cynthia Woods Mitchell Institute for Fundamental Physics and Astronomy, Texas A\&M University, College Station, TX 77843-4242, USA \\ ${ }^{3}$ International Centre for Radio Astronomy Research, The University of Western Australia, 35 Stirling Highway, \\ CRAWLEY WA 6009, Australia; martin.meyer@uwa.edu.au \\ ${ }^{4}$ Department of Astronomy, University of Massachusetts, Amherst, MA 01003, USA; calzetti@ astro.umass.edu \\ ${ }^{5}$ Illumina, Inc., 25861 Industrial Blvd, Hayward, CA 94545, USA; jharris@30doradus.org \\ Received 2012 March 29; accepted 2012 September 8; published 2012 November 15
}

\begin{abstract}
Dissolving stellar groups are very difficult to detect using traditional surface photometry techniques. We have developed a method to find and characterize non-compact stellar systems in galaxies where the young stellar population can be spatially resolved. By carrying out photometry on individual stars, we are able to separate the luminous blue stellar population from the star field background. The locations of these stars are used to identify groups by applying the HOP algorithm, which are then characterized using color-magnitude and stellar density radial profiles to estimate age, size, density, and shape. We test the method on Hubble Space Telescope Advanced Camera for Surveys archival images of IC 2574 and find 75 dispersed stellar groups. Of these, 20 highly dispersed groups are good candidates for dissolving systems. We find few compact systems with evidence of dissolution, potentially indicating that star formation in this galaxy occurs mostly in unbound clusters or groups. These systems indicate that the dispersion rate of groups and clusters in IC 2574 is at most $0.45 \mathrm{pc} \mathrm{Myr}^{-1}$. The location of the groups found with HOP correlate well with H I contour map features. However, they do not coincide with H I holes, suggesting that those holes were not created by star-forming regions.
\end{abstract}

Key words: galaxies: individual (IC 2574) - galaxies: star clusters: general - galaxies: stellar content - stars: early-type

Online-only material: color figures, extended figure, figure set

\section{INTRODUCTION}

The evolution of star clusters and other coeval stellar groups is a fundamental component of galaxy evolution. Their disruption and dissolution are crucial to build up the diffuse field star population in galaxies. Major efforts have been made to better understand the formation (e.g., Lada \& Lada 2003; Dale et al. 2005; Bonnell \& Bate 2006; Bate 2009; Girichidis et al. 2011) and evolution (e.g., Whitmore et al. 1999; Larsen 2004; Fall et al. 2005; Gieles et al. 2005; Bastian et al. 2009, 2012; Chandar et al. 2010, 2011; Karl et al. 2011) of star clusters. However, despite much theoretical work on their disruption (e.g., Fall \& Zhang 2001; Baumgardt \& Makino 2003; Lamers et al. 2005; Goodwin \& Bastian 2006; Gieles et al. 2006; Elmegreen \& Hunter 2010; Kruijssen et al. 2011), we have few observational constraints on the physical processes that lead to cluster and group disruption, the timescales on which they operate, and their environmental dependencies.

Existing observational studies suggest that the majority of star clusters may be destroyed within the first few $\times 10^{7} \mathrm{yr}$ of their life (e.g., Tremonti et al. 2001; Fall et al. 2005, 2009; Chandar et al. 2006, 2010). The destruction and dissolution processes are proposed to be first due to the tremendous loss of gas and dust during evolution of the most massive stars and their explosion in supernovae, although the efficiency of this phase to disrupt clusters has recently been challenged (see review by Bastian 2011). A recent theoretical work by Kruijssen et al. (2012) even suggests that gas removal by massive star evolution may not be the main player in the earlier disruption of star clusters, and the

\footnotetext{
6 Department of Physics, Mount Allison University, 67 York Street, Sackville,
} New Brunswick E4L 1E6, Canada. action of external tidal forces is proposed as another important process. Later on, external forces (e.g., tidal stripping, molecular cloud encounters), evaporation due to internal relaxation, as well as stellar evolution (e.g., Fellhauer \& Kroupa 2005; Bastian \& Goodwin 2006; Fall et al. 2009) are thought to play a role in cluster disruption.

Most empirical studies are based on counting clusters and groups still compact enough to provide sufficient contrast with the diffuse field population using surface photometry techniques. They do not tell us, for example, how stars from the remaining unbound clusters are distributed or how long it takes for them to dissolve into the diffuse field population. The recent work of Bastian et al. (2011), using a resolved stellar population, demonstrated the quick evolution of the global stellar structures, but again, it is not directly tackling the issue of identifying dissolving systems. Too much information is still missing to robustly characterize the last evolutionary stage: the actual disruption and dissolution of the cluster or group.

Since most of the original stellar content of clusters and coeval groups ends up in the diffuse field population, a complete understanding of disruption and dissolution processes must include highly dispersed populations. In this work, we present a new method which uses observations of resolved stellar populations to identify young coeval stellar groups that are spatially too dispersed to be easily detectable with traditional surface photometric techniques. We then characterize these groups to find suitable candidates for dissolving star clusters. The ultimate goal is to better understand how star clusters and groups disrupt and dissolve in the context of various environmental parameters such as the potential well, the radial position, the influence of gravitational instabilities, interaction and mergers, and the star formation intensity. 


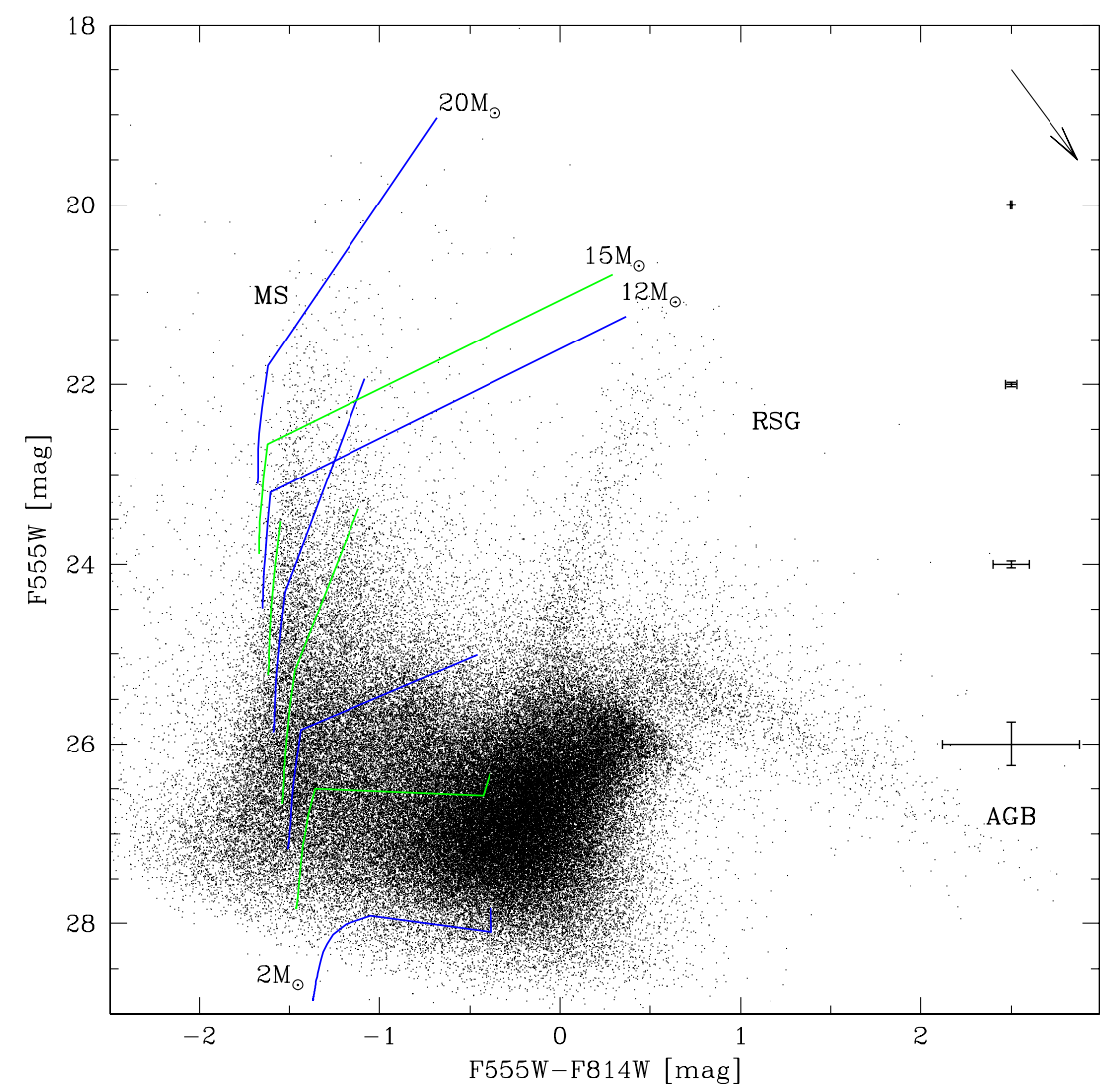

Figure 1. Stellar color-magnitude diagram (F555W and F814W) of IC 2574. Alternating blue and green lines are Padova stellar evolutionary tracks for stars with initial masses of 2, 3, 4, 5, 7, 9, 12,15, and $20 M_{\odot}$, where only a few are labeled for clarity. Main evolutionary features for the main sequence (MS), the red supergiant branch (RSG), and the asymptotic giant branch (AGB) are indicated. The 1 mag absorption vector in the F555W filter is shown in the upper right corner. Mean photometric uncertainties are indicated by crosses on the right for their corresponding magnitude bins.

(A color version of this figure is available in the online journal.)

In this work, we study IC 2574, a gas rich dwarf irregular disk galaxy located 3.8 Mpc away (Dalcanton et al. 2009) and part of the M 81 interacting group. It has a dynamical mass of $8.3 \times 10^{9} M_{\odot}$ and displays a solid body rotation curve (Walter \& Brinks 1999) that minimizes the effect of radial shears that could theoretically accelerate cluster disruption. The galaxy has a global average star formation rate (SFR) of $8.63 \times$ $10^{-2} M_{\odot} \mathrm{yr}^{-1}$ over the Hubble time and its SFR is currently above the global average, although it was even higher $500 \mathrm{Myr}$ to 1 Gyr ago (Weisz et al. 2008). This recent high SFR over the past Gyr makes this galaxy a good target for the search of young star clusters and aggregates. IC 2574 hosts many $\mathrm{H} \mathrm{I}$ holes, including a supergiant shell (SGS) of $\sim 1000 \times 500 \mathrm{pc}$ in deprojected size (Walter et al. 1998; Walter \& Brinks 1999; Cannon et al. 2005). The SGS, as well as many holes found in IC 2574, is surrounded with $\mathrm{H}$ II regions likely triggered by the stellar feedback related to the star-forming event that occurred in the hole center $\sim 25 \mathrm{Myr}$ ago (Walter \& Brinks 1999; Weisz et al. 2009). Beside some star-forming regions surrounding the SGS and a few globular clusters (Georgiev et al. 1996), the star cluster population in IC 2574 is not well studied. Its metallicity is fairly low, with $12+\log [\mathrm{O} / \mathrm{H}]=7.93 \pm 0.05$ (Croxall et al. 2009).

\section{DATA REDUCTION AND PHOTOMETRY}

Archival images from the Advanced Camera for Surveys (ACS) on board the Hubble Space Telescope (HST) were downloaded from the Multimission Archive at STScI (MAST ${ }^{7}$ ). This galaxy was chosen because of its recent star formation and the depth of available images. Observations were originally taken on 2004 February 6 for the cycle 12 project ID 9755 (PI: F. Walter) in the F435W, F555W, F814W, and F658N filters (roughly $B, V, I$, and $\mathrm{H} \alpha$ ). The field of view includes the SGS and its triggered star formation sites, as well as another strong $\mathrm{H}$ II region. The data were reduced by the STScI calibration pipeline, which includes a treatment for bias, flat, cosmic rays, and drizzling. ${ }^{8}$ For IC 2574 , the spatial scale is $0.9 \mathrm{pc} \mathrm{pixel}^{-1}$ $\left(0{ }^{\prime} .05\right.$ pixel $\left.^{-1}\right)$ and the spatial resolution is about $1.8 \mathrm{pc}$ (PSF FWHM of 2.1, 2.0, and 2.0 pixels for the F435W, F555W, and F814W images, respectively), which allows us to resolve individual stars except for compact clusters where crowding is severe.

We performed point-spread function (PSF) photometry on the F435W, F555W, and F814W images using the IRAF/DAOPHOT package. We selected $\sim 100$ bright unsaturated stars to create a PSF model with the form of a Moffat function having a 15 pixels radius. Note that the PSF varies slightly within the ACS field of view, contributing to photometric uncertainties. The photometric calibration was performed using the STMAG zero points by Sirianni et al. (2005). The color-magnitude diagram (CMD) for the stars found in IC 2574 is shown in Figure 1. We calculated aperture corrections of

\footnotetext{
7 http://archive.stsci.edu/

8 See http://www.stsci.edu/hst/acs/analysis/.
} 
$0.182,0.215$, and 0.242 mag for the F435W, F555W, and F814W filters, respectively. We performed an artificial star test and found that the $50 \%$ detection limits are reached at 27.7, 26.8, and $28.7 \mathrm{mag}$ for the same filters, respectively. Figure 1 compares these limits to stellar evolutionary tracks from the Padova group, ${ }^{9}$ indicating that we detect few stars less massive than 2 or $3 M_{\odot}$. We corrected for a homogeneous foreground Galactic extinction of $E(B-V)=0.036$ mag (Schlegel et al. 1998).

\section{THE RESOLVED STAR APPROACH TO STUDY STELLAR GROUPS}

With the goal of retrieving young dispersed stellar groups, we have developed a method to identify potentially dissolving overdensities of young stars in nearby extragalactic systems, as detailed below.

1. We first select a nearby galaxy with resolved individual O- and B-type stars. Galaxies with young stellar populations are targeted because these stars are good tracers of young star clusters or groups, such as those that might dissolve in great numbers in the first few $10^{8} \mathrm{yr}$ of their life (Fall et al. 2005). Such clusters and groups should still host relatively massive O- and/or B-type stars, if they were produced. Also, these stars are bright, and therefore easier to separate from the diffuse field population of previous generations of stars. Finally, we have a better handle on their age since they evolve rapidly. The approach requires nearby galaxies ( $\leqslant 5 \mathrm{Mpc}$ with $H S T$ ) to resolve their stellar content. We avoid galaxies, including our own, that are too inclined to clearly study their stellar content due to crowding and blending effects or interstellar absorption. Ideally, multiband imaging is used (including the $U$ band, although not available in this work) to better differentiate between the most massive stars (Anders et al. 2004) and ultimately derive a more accurate age for each stellar group found.

2. We extract the stellar photometric information as described in Section 2. Using a CMD, we identify bright blue massive hot stars. For IC 2574, we used the criteria F555W $\leqslant 25.0$ mag and F555W-F814W $\leqslant-0.44$ mag. This roughly correspond to stars with initial masses of $5 M_{\odot}$ and higher, or B-type stars. These cuts exclude stars with typically large photometric uncertainties and include stars that have a greater chance to stand out against the diffuse older stellar population.

3. The next step is to find overdensities within the selected sample of bright blue stars. We make use of a group-finding algorithm, HOP, developed by Eisenstein \& Hut (1998). This algorithm was created originally for cosmological and galaxy evolution studies. It is a sophisticated version of friends-of-friends algorithms. It is based on the spatial density of particles, where each particle is grouped with its neighbor that has the highest density, which is not necessarily the nearest neighbor. For each particle, a density is assigned by smoothing over a chosen number of particles. The groups are formed based on a saddle density value used to separate each group, a maximum density value for a group to be viable and not merged with another, and a minimum density value for a particle to be a member of a group. Maximum, minimum, and saddle density values are assigned by the user. This allows us to find groups within groups, as one would expect in a hierarchical star formation

\footnotetext{
9 http://pleiadi.pd.astro.it
}

Table 1

Parameter Grid Explored with HOP

\begin{tabular}{ll}
\hline \hline Parameter $^{\mathrm{a}}$ & \multicolumn{1}{c}{ Parameter Values } \\
\hline$d$ & $1,2,3,4,6$ \\
$h$ & $6,9,16,32$ \\
$o$ & $10,100,1000,10000$ \\
$p$ & $2000,10000,25000,50000$ \\
$s$ & $500,700,1000,1500$ \\
$m$ & $2,5,10$ \\
\hline
\end{tabular}

Note. ${ }^{\text {a }} d$ is the number of particles to smooth for the density calculation, $h$ is the number of neighbors to look at when searching the highest density neighbor, $o$ is the minimum density required for a particle to be in a group, $p$ is the minimum central density needed for a group to be viable, $s$ is the boundary value needed for two groups to be merged, and $m$ is the minimum number of particles required to form a group.

scenario (e.g., Efremov 1995; Efremov \& Elmegreen 1998; Elmegreen et al. 2000; Elmegreen 2002, 2011; Elmegreen $\&$ Hunter 2010 and reference therein). For this work, we run HOP with a full grid of density values, which are reported in Table 1 together with a description of the six parameters. We then visually examine the grouping results of different parameter runs to find multiply identified locations. We generally obtain better results when using a small number of particles $d$ to smooth over ( $d=1$ or 2 ) and a relatively low number of neighbors $h$ to look at when searching for the highest density neighbor ( $h=6$ or 9$)$. Smaller values of $o$, the minimum density value for a particle to be in a group, are better at finding sparse groups or groups in low stellar density environments, while large values of $o$ are more successful at picking up groups in and around intense star-forming regions. Only groups found in multiple analyses (i.e., with various grid parameters) are considered for further investigation.

4. We estimate the radial density and size of each stellar group found with HOP. We make a preliminary ellipse fit using only the bright blue stars that form the group. Then, we include the full photometric list of stars, including the fainter ones, within the ellipse. Using the fitted ellipse, we calculate the surface density of stars within concentric ellipses determined based on the ellipse size. This creates a radial density profile, with the radius defined along the semimajor axis, which provides an estimate of the local diffuse field density. We define the size of the group where the density level flattens. This method may introduce an environmental bias where the group size tends to be smaller in denser background populations. Examples of radial density profiles are shown in Figures 2(a)-(i). Note that the stellar density values are limited to the photometric detection limits of the images used.

A visual inspection of the images and the ellipse fits indicates that in most cases, the ellipse fits based on the bright blue stars are not representative of the whole stellar group. This is due to the fact that massive stars are relatively rare and are not found everywhere within the stellar group. Therefore, massive stars are not good tracers of the physical shape of groups. Unlike compact star clusters, stellar groups identified in this work do not always have their maximum density at the center due to irregular stellar 

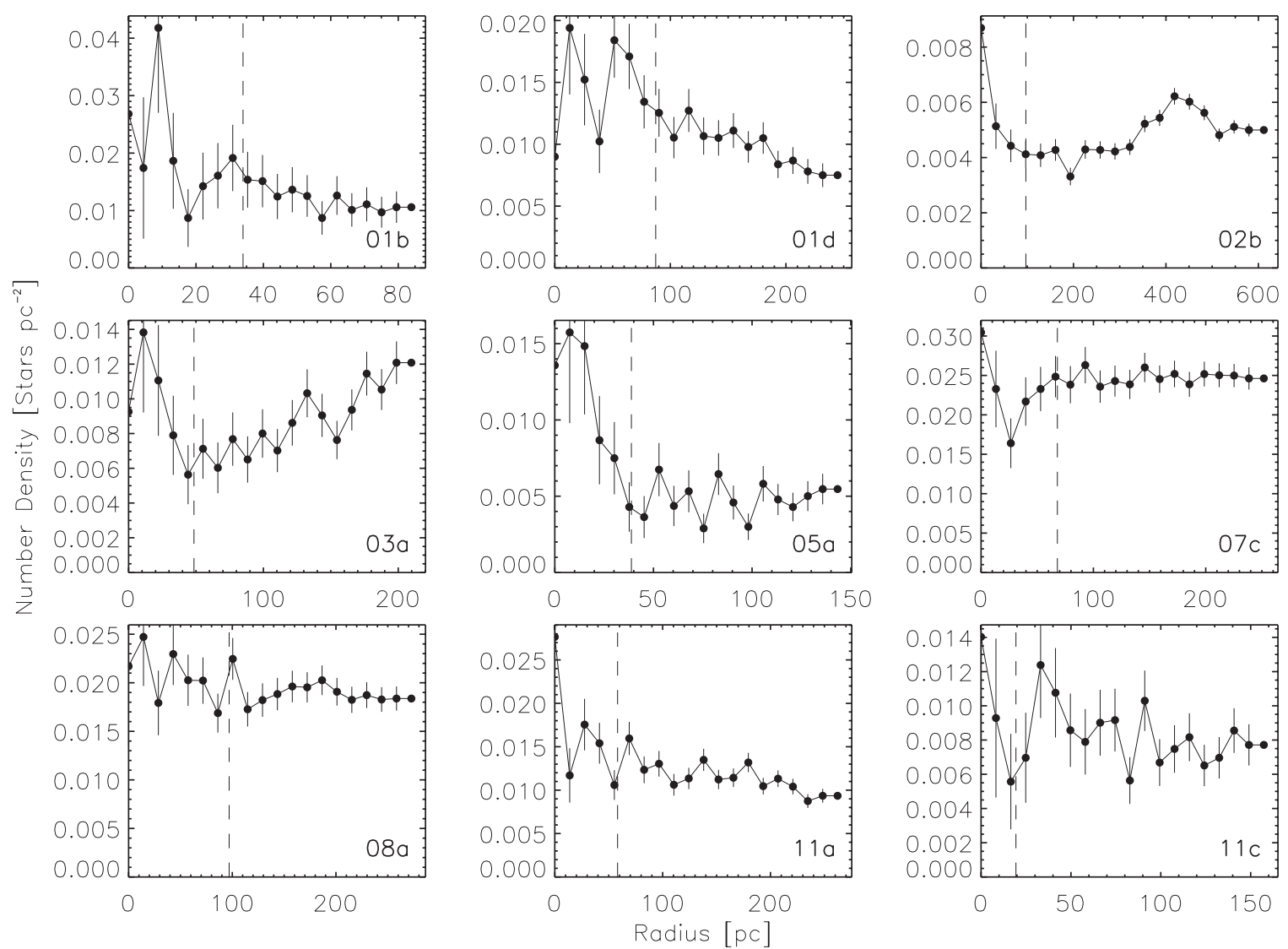

Figure 2. Radial density profiles for stellar groups found with HOP. The radii are given along the semimajor axis of the fitted ellipse. The vertical dashed lines indicate the approximate size of each group as the location where the stellar density reaches the diffuse field level. Groups are identified on the lower right corner of each panel. All radial density profiles are available online.

(An extended version of this figure is available in the online journal.)

distribution within the group. This is to be expected for sparse systems.

5. We plot the stellar CMD of each group within the ellipse size determined with the density profile to verify if the group is consistent with a coeval single stellar population. We check both F555W-F814W versus F555W and $\mathrm{F} 435 \mathrm{~W}-\mathrm{F} 814 \mathrm{~W}$ versus $\mathrm{F} 435 \mathrm{~W}$ diagrams for a consistency check and more robust results. The diagram using the F435W-F814W color offers a more accurate age estimate than the F555W-F814W for massive stars, and the F435W filter is also less affected by nebular emission, F435W excluding the [O III] $\lambda \lambda$ 4959,5007 doublet. For each group, we compare the given CMD with isochrones from the Padova group and kindly provided specifically for the ACS bandpasses. We discard groups with stars clearly inconsistent with a coeval system, although we allow for some discrepancies due to photometric errors, interstellar reddening, nebular contamination, and poor statistical filling. We estimate an age for each cluster based on a visual fit with the isochrones. Note that for IC 2574, these ages are not as accurate as they would be if we had $U$-band photometry. However, we can still roughly estimate age to within a few $10^{7} \mathrm{yr}$, and we can still accurately date groups younger than $10 \mathrm{Myr}$ due to their associated nebular emission generated only by ionizing stars and detected as diffuse emission in the $\mathrm{H} \alpha$ image. Some nebular emission can also be detected in the other filters since they include other strong nebular lines (e.g., the F555W filter includes the O [III] $\lambda \lambda 4959,5007$ doublet) and nebular continuum. For the isochrone fits, we disregard a region within the CMD where stars are likely to come from many different generations of stars, and unlikely to be part of a young coeval stellar group. The region includes stars fainter than $25.0 \mathrm{mag}$ in the F555W filter and redder than -1.0 in the F555W-F814W color. For the purpose of this work, this region is considered to be populated by a diffuse field population.

We considered deriving a stellar mass for each stellar group, but unfortunately the age uncertainties associated with the sparse mass function sampling do not allow a reliable mass estimate for the groups.

\section{STELLAR GROUPS FOUND WITH HOP}

We identified 75 likely coeval stellar groups using the method described in Section 3 (overlaid on F555W image in Figure 3). We divide these groups into three main categories. The first (hereafter category I) includes groups with significant nebular emission. The list of category I groups is given in Table 2 . Although the detection of such stellar systems is not the aim of the method developed in this work, it is reassuring that all groups with nebular emission are easily detected with HOP. In this category, the stellar groups can usually be identified visually due to their compactness and the bright gaseous emission surrounding them. The nebular gas is more prominent in the F555W and F658N filters which include the $[\mathrm{O} \mathrm{III}]+\mathrm{H} \beta$ and the $\mathrm{H} \alpha$ nebular lines, respectively. For these groups, it is fairly easy and reliable to estimate an age even in cases where only a very few stars constrain the isochrone fitting on the CMD. 


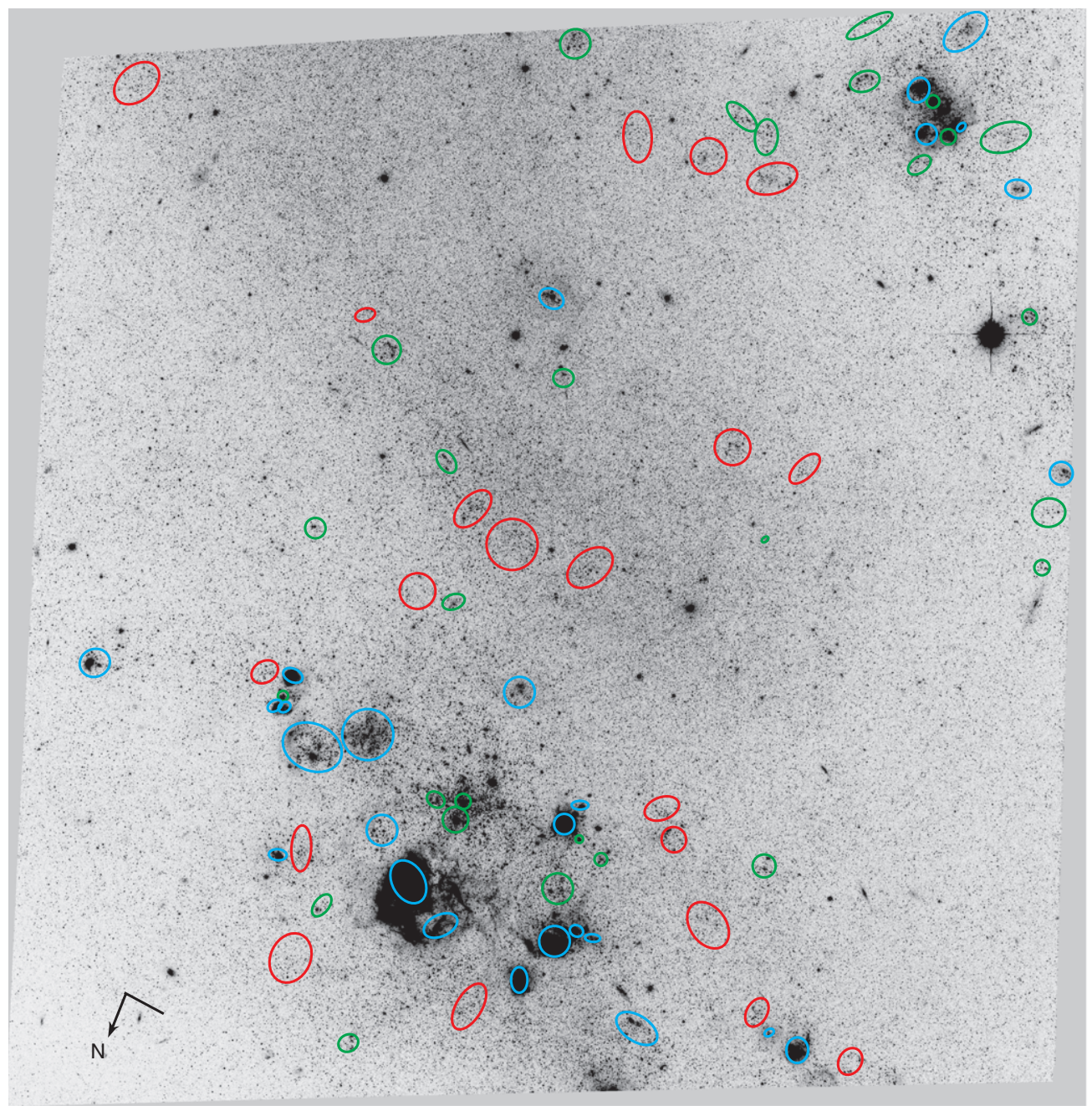

Figure 3. Stellar groups found with HOP and overlaid on the F555W HST/ACS image. The ellipses show the fitted size and shape of each group, and defined as the location where the stellar density reaches the background level. Blue ellipses indicate category I groups while green ellipses indicate category II groups and red show category III groups. The image is about $3.9 \times 3.9 \mathrm{kpc}^{2}$. The northwest directions are indicated in the lower left.

Given that ionizing photons are needed to create these emission lines, which are only produced by the most massive OB stars, the presence of nebular emission indicates an age younger than $\sim 10$ Myr. This first category includes extended unbound starforming systems such as small $\mathrm{OB}$ associations and OB groups. For category I groups, the diffuse field density level can be difficult to estimate because the density profile tends to slowly declines over an extended radius and does not really flatten. This usually occurs when the stellar group is associated with an extended population of diffuse blue stars.

The second category (category II) includes groups with a stellar overdensity that contrasts well with the surrounding diffuse field population, and a CMD consistent with a coeval population. The majority of these groups would not be detected by traditional surface photometry techniques but can be identified with a careful visual inspection of our HST images. Those groups, reported in Table 3, do not show significant nebular emission.

The third category (or category III) contains very sparse groups that would certainly have been missed using traditional surface photometry identification methods. They mainly differ from category II groups in that their radial stellar densities show marginal enhancements compared to the surrounding diffuse field, if any. However, their CMD usually shows a few stars consistent with an isochrone that separates well from the diffuse field population. The list of such groups is provided in Table 4 . Although most of those groups may be asterisms, they are good candidates for advanced dissolving stellar systems. Keep in mind that our dissolving candidates do not necessarily originate from a compact cluster or group with a size of only a few parsecs, but can also be the remnants of loose, larger systems found in complexes, such as OB associations and OB groups, like those we found for category I groups.

Detailed notes on each individual stellar group are reported in the Appendix. For each group, the HST/ACS image, CMD, and ellipse fitting are reported in Figure 4.

\subsection{Global Properties of Stellar Groups}

We find that the median sizes and ages are as follows: $50 \mathrm{pc}$ and $10 \mathrm{Myr}$ for category I groups with nebular emission; $45 \mathrm{pc}$ and $30 \mathrm{Myr}$ for category II groups; and $90 \mathrm{pc}$ and $40 \mathrm{Myr}$ for the highly dispersed systems of category III. The median ages are often based on upper limits, as indicated in Tables 2-4, and are likely to be younger.

It is interesting to note the similar sizes found for category I and II groups. If category II groups are more evolved versions of category I groups, then we might expect category II groups to be larger on average. The fact that the observed cluster sizes 
Table 2

Physical Properties of Stellar Groups with Nebular Emission (Category I)

\begin{tabular}{|c|c|c|c|c|c|c|c|c|c|}
\hline ID & $\begin{array}{l}\text { X-center } \\
\text { (pixel) }\end{array}$ & $\begin{array}{l}\text { Y-center } \\
\text { (pixel) }\end{array}$ & $\alpha(\mathrm{J} 2000)$ & $\delta(\mathrm{J} 2000)$ & $\epsilon$ & $\begin{array}{l}\text { P.A. } \\
\text { (deg) }\end{array}$ & $\begin{array}{l}\text { Size } \\
(\mathrm{pc})\end{array}$ & $\begin{array}{l}\text { Background } \\
\left(\text { stars } \mathrm{pc}^{-2}\right)\end{array}$ & $\begin{array}{l}\text { Age }^{\mathrm{a}} \\
(\mathrm{Myr})\end{array}$ \\
\hline $01 b$ & 1062 & 980 & $10: 28: 39.225$ & $+68: 28: 30.70$ & 0.781 & -5 & $25-35$ & 0.01 & $<10$ \\
\hline $01 z$ & 1197 & 1401 & $10: 28: 38.949$ & $+68: 28: 08.65$ & 0.652 & 155 & 120 & 0.007 & $10-15$ \\
\hline $02 \mathrm{z}$ & 1414 & 1451 & $10: 28: 40.612$ & $+68: 28: 02.31$ & 0.000 & 0 & 100 & 0.014 & $\sim 10+40$ \\
\hline $03 z$ & 1470 & 1077 & $10: 28: 42.342$ & $+68: 28: 18.64$ & 0.000 & 0 & 60 & 0.012 & $\sim 40$ \\
\hline $07 \mathrm{c}$ & 1699 & 703 & $10: 28: 45.533$ & $+68: 28: 31.78$ & 0.802 & 25 & 70 & 0.025 & $\leqslant 15$ \\
\hline $13 \mathrm{a}$ & 3095 & 215 & $10: 28: 58.955$ & $+68: 28: 28.56$ & 0.515 & -92 & 50 & 0.01 & $<10$ \\
\hline $13 z$ & 1573 & 874 & $10: 28: 43.894$ & $+68: 28: 26.17$ & 0.719 & 120 & 90 & 0.016 & $<10$ \\
\hline $15 \mathrm{z}$ & 2183 & 1100 & $10: 28: 48.280$ & $+68: 28: 04.37$ & 0.000 & 0 & 40 & 0.013 & $<10$ \\
\hline $18 \mathrm{c}$ & 2467 & 300 & $10: 28: 53.370$ & $+68: 28: 36.26$ & 0.831 & 147 & 90 & 0.017 & $20-40$ \\
\hline $21 z$ & 2145 & 642 & $10: 28: 49.501$ & $+68: 28: 26.35$ & 0.000 & 0 & 60 & 0.015 & $<10$ \\
\hline $22 \mathrm{z}$ & 2231 & 682 & $10: 28: 50.092$ & $+68: 28: 22.90$ & 0.600 & 155 & 27 & 0.013 & $<10$ \\
\hline $23 \mathrm{c}$ & 346 & 1731 & $10: 28: 30.660$ & $+68: 28: 09.04$ & 0.426 & 23 & $60-100$ & 0.001 & $<10$ \\
\hline $24 \mathrm{z}$ & 2007 & 490 & $10: 28: 48.849$ & $+68: 28: 35.96$ & 0.768 & 90 & 50 & 0.01 & $<10$ \\
\hline $25 \mathrm{c}$ & 1118 & 1676 & $10: 28: 37.359$ & $+68: 27: 57.33$ & 0.745 & -25 & 40 & 0.006 & $<10$ \\
\hline $25 \mathrm{e}$ & 1050 & 1563 & $10: 28: 37.165$ & $+68: 28: 03.84$ & 0.724 & 32 & $10-40$ & 0.008 & $<10$ \\
\hline $25 \mathrm{~g}$ & 1088 & 1558 & $10: 28: 37.502$ & $+68: 28: 03.37$ & 0.650 & 30 & 25 & 0.005 & $<10$ \\
\hline $28 b$ & 2008 & 1616 & $10: 28: 45.068$ & $+68: 27: 43.65$ & 0.000 & 0 & 60 & 0.012 & $\leqslant 10$ \\
\hline $29 f$ & 2245 & 1174 & $10: 28: 48.554$ & $+68: 27: 59.79$ & 0.857 & 0 & 33 & 0.015 & $<10$ \\
\hline $50 \mathrm{z}$ & 3570 & 3973 & $10: 28: 50.308$ & $+68: 25: 25.25$ & 0.578 & 65 & 50 & 0.011 & $<10$ \\
\hline $52 z$ & 3737 & 3828 & $10: 28: 52.201$ & $+68: 25: 28.89$ & 0.768 & 45 & 20 & 0.01 & $<10$ \\
\hline $54 z$ & 3601 & 3800 & $10: 28: 51.150$ & $+68: 25: 32.71$ & 0.000 & 0 & 40 & 0.012 & $<15$ \\
\hline $56 a$ & 4128 & 2473 & 10:29:00.052 & $+68: 26: 24.55$ & 0.000 & 0 & 45 (60?) & 0.001 & $<15$ \\
\hline $74 \mathrm{c}$ & 3959 & 3586 & $10: 28: 54.884$ & $+68: 25: 36.01$ & 0.690 & 172 & $50-60$ & 0.03 & $<10$ \\
\hline $75 \mathrm{e}$ & 3753 & 4201 & $10: 28: 51.082$ & $+68: 25: 11.27$ & 0.829 & 40 & 100 & 0.006 & $<10$ \\
\hline $83 d$ & 2133 & 3157 & $10: 28: 40.945$ & $+68: 26: 29.76$ & 0.670 & 151 & $50-65$ & 0.021 & $<10$ \\
\hline
\end{tabular}

Note. ${ }^{\text {a }}$ Ages are rough estimates based on the CMDs and Padova isochrones.

Table 3

Physical Properties of Stellar Groups found with HOP (Category II)

\begin{tabular}{|c|c|c|c|c|c|c|c|c|c|}
\hline ID & $\begin{array}{l}\text { X-center } \\
\text { (pixel) }\end{array}$ & $\begin{array}{l}\text { Y-center } \\
\text { (pixel) }\end{array}$ & $\alpha(\mathrm{J} 2000)$ & $\delta(\mathrm{J} 2000)$ & $\epsilon$ & $\begin{array}{l}\text { P.A. } \\
\text { (deg) }\end{array}$ & $\begin{array}{l}\text { Size } \\
(\mathrm{pc})\end{array}$ & $\begin{array}{l}\text { Background } \\
\left(\text { stars } \mathrm{pc}^{-2}\right)\end{array}$ & $\begin{array}{l}\text { Age }^{\mathrm{a}} \\
(\mathrm{Myr})\end{array}$ \\
\hline $03 \mathrm{a}$ & 1234 & 782 & $10: 28: 41.342$ & $+68: 28: 36.71$ & 0.824 & 52 & $40-60$ & 0.006 & $\sim 40$ \\
\hline $05 a$ & 1338 & 243 & $10: 28: 44.033$ & $+68: 28: 59.83$ & 0.559 & 25 & 40 & 0.004 & $10-15$ \\
\hline $05 z$ & 1757 & 1117 & $10: 28: 44.629$ & $+68: 28: 11.47$ & 0.000 & 0 & 50 & 0.017 & $15-20$ \\
\hline $06 z$ & 1680 & 1196 & $10: 28: 43.714$ & $+68: 28: 09.23$ & 0.621 & 145 & 37 & 0.017 & $30-40$ \\
\hline $18 z$ & 2242 & 1041 & $10: 28: 48.976$ & $+68: 28: 06.02$ & 0.000 & 0 & 15 & 0.005 & $<20$ \\
\hline $19 z$ & 2327 & 962 & $10: 28: 49.959$ & $+68: 28: 08.11$ & 0.000 & 0 & 25 & 0.012 & $\leqslant 15$ \\
\hline $20 z$ & 2158 & 848 & $10: 28: 48.917$ & $+68: 28: 16.54$ & 0.000 & 0 & 60 & 0.017 & $10-15$ \\
\hline $25 \mathrm{~h}$ & 1082 & 1602 & $10: 28: 37.304$ & $+68: 28: 01.44$ & 0.000 & 0 & $20-40$ & 0.008 & $10-15$ \\
\hline $34 b$ & 1209 & 2259 & $10: 28: 36.169$ & $+68: 27: 28.57$ & 0.000 & 0 & 40 & 0.004 & $40 ?$ \\
\hline $40 \mathrm{~b}$ & 1489 & 2956 & $10: 28: 36.191$ & $+68: 26: 51.01$ & 0.000 & 0 & 55 & 0.013 & $\leqslant 15$ \\
\hline $41 \mathrm{a}$ & 1722 & 2519 & $10: 28: 39.622$ & $+68: 27: 07.00$ & 0.771 & 125 & 50 & 0.017 & $\leqslant 40$ \\
\hline $51 z$ & 3626 & 3928 & $10: 28: 50.931$ & $+68: 25: 26.30$ & 0.000 & 0 & 25 & 0.015 & $10-15$ \\
\hline $53 z$ & 3685 & 3790 & $10: 28: 51.891$ & $+68: 25: 31.62$ & 0.000 & 0 & 30 & 0.012 & $10-15$ \\
\hline $54 c$ & 4052 & 2104 & $10: 29: 00.655$ & $+68: 26: 43.09$ & 0.000 & 0 & 30 & 0.002 & $\leqslant 70$ \\
\hline $55 \mathrm{~b}$ & 4079 & 2319 & $10: 29: 00.158$ & $+68: 26: 32.61$ & 0.515 & 7 & 65 & 0.002 & $\leqslant 20$ \\
\hline $58 \mathrm{a}$ & 2180 & 2846 & $10: 28: 42.385$ & $+68: 26: 43.34$ & 0.484 & 0 & 40 & 0.017 & $\leqslant 20$ \\
\hline $61 c$ & 2969 & 2214 & $10: 28: 51.159$ & $+68: 26: 58.07$ & 0.793 & 38 & $15-20$ & 0.015 & $\leqslant 40$ \\
\hline $69 \mathrm{e}$ & 2226 & 4154 & $10: 28: 38.383$ & $+68: 25: 41.73$ & 0.305 & 23 & 60 & 0.010 & $\leqslant 20$ \\
\hline $71 \mathrm{a}$ & 2878 & 3869 & $10: 28: 44.830$ & $+68: 25: 42.90$ & 0.898 & -44 & 75 & 0.010 & 40 \\
\hline $71 b$ & 2974 & 3789 & $10: 28: 45.908$ & $+68: 25: 44.84$ & 0.768 & 85 & 70 & 0.015 & 30 \\
\hline $72 b$ & 4004 & 3085 & $10: 28: 56.948$ & $+68: 25: 58.44$ & 0.338 & 115 & $30-50$ & 0.008 & $<10$ \\
\hline $76 \mathrm{~d}$ & 3359 & 4007 & $10: 28: 48.417$ & $+68: 25: 27.58$ & 0.760 & 18 & 60 & 0.010 & 40 \\
\hline $77 \mathrm{a}$ & 3573 & 3680 & $10: 28: 51.318$ & $+68: 25: 38.80$ & 0.798 & 35 & $50-70$ & 0.013 & 40 \\
\hline $80 c$ & 3911 & 3788 & $10: 28: 53.800$ & $+68: 25: 27.52$ & 0.821 & 15 & 100 & 0.07 & 30 \\
\hline $81 \mathrm{c}$ & 3378 & 4225 & $10: 28: 47.845$ & $+68: 25: 17.11$ & 0.959 & 28 & 100 & 0.010 & $\leqslant 40$ \\
\hline
\end{tabular}

Note. ${ }^{\text {a }}$ Ages are rough estimates based on the CMDs and Padova isochrones. 

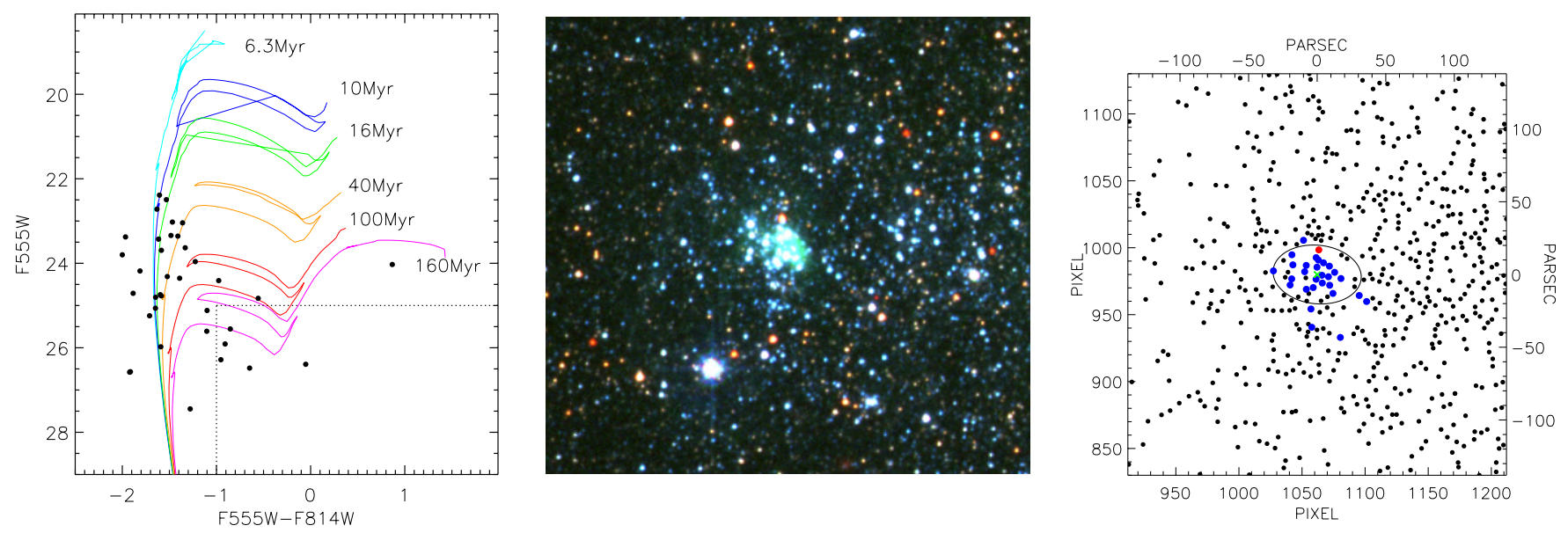

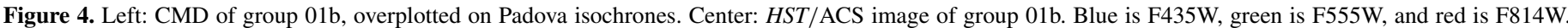

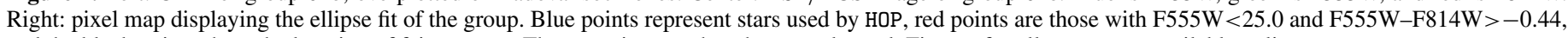
and the black points show the location of fainter stars. The map size matches the central panel. Figures for all groups are available online.

(The complete figure set (225 images) and a color version of this figure are available in the online journal.)

Table 4

Physical Properties of Highly Dispersed Stellar Groups (Category III)

\begin{tabular}{|c|c|c|c|c|c|c|c|c|c|}
\hline ID & $\begin{array}{l}\text { X-center } \\
\text { (pixel) }\end{array}$ & $\begin{array}{l}\text { Y-center } \\
\text { (pixel) }\end{array}$ & $\alpha(\mathrm{J} 2000)$ & $\delta(\mathrm{J} 2000)$ & $\epsilon$ & $\begin{array}{l}\text { P.A. } \\
\text { (deg) }\end{array}$ & $\begin{array}{l}\text { Size } \\
(\mathrm{pc})\end{array}$ & $\begin{array}{l}\text { Background } \\
\left(\text { stars } \mathrm{pc}^{-2}\right)\end{array}$ & $\begin{array}{l}\text { Age }^{\mathrm{a}} \\
(\mathrm{Myr})\end{array}$ \\
\hline $01 d$ & 1154 & 1005 & $10: 28: 39.917$ & $+68: 28: 27.84$ & 0.904 & 87 & 90 & 0.011 & $\leqslant 40$ \\
\hline $02 \mathrm{~b}$ & 1112 & 576 & $10: 28: 41.005$ & $+68: 28: 48.54$ & 0.626 & -115 & 100 & 0.004 & $40-100$ \\
\hline $08 \mathrm{a}$ & 1811 & 386 & $10: 28: 47.545$ & $+68: 28: 44.42$ & 0.866 & 59 & $100-120$ & 0.018 & $\leqslant 20-40$ \\
\hline $11 \mathrm{a}$ & 2937 & 363 & $10: 28: 57.123$ & $+68: 28: 24.62$ & 0.745 & 60 & $60-70$ & 0.011 & $<30$ \\
\hline $15 \mathrm{a}$ & 3302 & 171 & 10:29:00.849 & $+68: 28: 26.76$ & 0.595 & 58 & 55 & 0.004 & 40 \\
\hline $25 \mathrm{i}$ & 1011 & 1696 & $10: 28: 36.389$ & $+68: 27: 58.38$ & 0.656 & 30 & 55 & 0.004 & 40 \\
\hline $30 \mathrm{~b}$ & 2612 & 1039 & $10: 28: 52.104$ & $+68: 27: 59.26$ & 0.289 & 110 & 50 & 0.012 & $10-40$ \\
\hline $30 d$ & 2565 & 1161 & $10: 28: 51.297$ & $+68: 27: 54.46$ & 0.780 & 20 & $70-90$ & 0.008 & $\sim 100$ \\
\hline $38 \mathrm{c}$ & 1825 & 2334 & $10: 28: 41.112$ & $+68: 27: 13.69$ & 0.831 & 45 & 90 & 0.011 & $10-40$ \\
\hline $47 b$ & 1610 & 2012 & $10: 28: 40.381$ & $+68: 27: 32.62$ & 0.000 & 0 & 70 & 0.009 & $\leqslant 20$ \\
\hline $49 a$ & 1979 & 2195 & $10: 28: 42.878$ & $+68: 27: 17.29$ & 0.000 & 0 & 100 & 0.013 & $\sim 100$ \\
\hline $51 \mathrm{a}$ & 2842 & 2575 & $10: 28: 48.875$ & $+68: 26: 43.66$ & 0.000 & 0 & 70 & 0.012 & $15-100$ \\
\hline $60 \mathrm{e}$ & 3124 & 2491 & $10: 28: 51.534$ & $+68: 26: 42.34$ & 0.872 & 44 & $60-90$ & 0.017 & $40-100$ \\
\hline $62 c$ & 510 & 4000 & $10: 28: 24.439$ & $+68: 26: 20.58$ & 0.732 & 40 & 100 & 0.007 & 40 \\
\hline $67 d$ & 1404 & 3093 & $10: 28: 35.015$ & $+68: 26: 46.22$ & 0.771 & 15 & $40-65$ & 0.015 & 40 \\
\hline $70 \mathrm{~b}$ & 2470 & 3790 & $10: 28: 41.659$ & $+68: 25: 54.12$ & 0.883 & 93 & 100 & 0.009 & 100 \\
\hline $71 \mathrm{c}$ & 2997 & 3626 & $10: 28: 46.649$ & $+68: 25: 51.98$ & 0.809 & 15 & 100 & 0.01 & $\leqslant 15$ \\
\hline $71 \mathrm{e}$ & 2748 & 3714 & $10: 28: 44.256$ & $+68: 25: 52.51$ & 0.000 & 0 & 70 & 0.011 & 60 \\
\hline
\end{tabular}

Note. ${ }^{\text {a }}$ Ages are rough estimates based on the CMDs and Padova isochrones.

are similar suggests that either the evolutionary antecedents of category II groups were actually more compact than the observed category I groups, or that the dispersing mechanisms acting in IC 2574 do not have a significant effect over a 20-30 Myr timescale. As already pointed out by Weisz et al. (2009), IC 2574 is a dwarf irregular galaxy that behaves more like a solid body rotator. In other words, IC 2574 offers essentially no differential rotation and limited sheer forces (other than those created by its interaction with M 81) that could accelerate the dispersion of the groups through tidal shocks, as recently modeled nicely by Kruijssen et al. (2011).

In Figure 5, we look for a possible correlation between the size of the groups and their age, assuming that all groups are not asterisms. Although we are dealing with a small size sample with fairly large uncertainties, the figure indicates a clear trend for older clusters to be larger in size, with a low dispersion rate of $0.45 \mathrm{pc} \mathrm{Myr}^{-1}$. This trend is more consistent with the scenario in which external forces capable of disrupting stellar groups and clusters are weak in IC 2574. Based on Figure 3, we do not see any correlation between the position of the groups and the position angle of the fitted ellipses. This is also consistent with the absence of differential rotation and sheer of the dwarf irregular galaxy.

Assuming that the groups were originally compact, we find that the median group dispersion speeds are $3.9 \pm 2.7,1 \pm 1$, and $1 \pm 1 \mathrm{~km} \mathrm{~s}^{-1}$ for groups in categories I, II, and III, respectively. Those are very plausible low values considering that the groups were probably not compact at birth and that these values are upper limits only.

\section{POSSIBLE PHYSICALLY RELATED GROUPS}

Some stellar groups reported in Tables 2-4 are located near to each other and may be physically linked by a common 


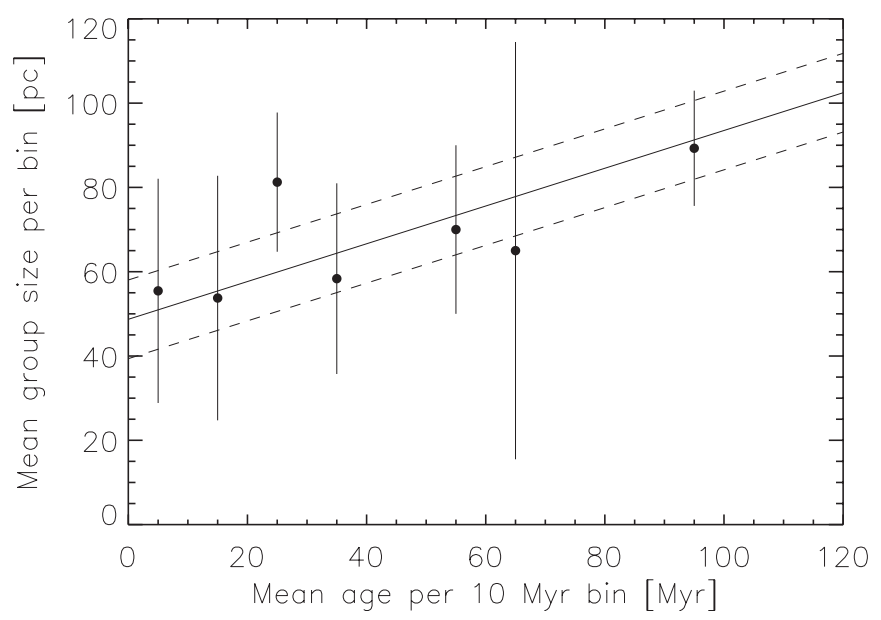

Figure 5. Mean size of groups as a function of their age. The line is the best linear ordinary least-square fit, with the $3 \sigma$ deviation indicated by dashed lines.

star-forming event and be part of larger size systems, as described by Efremov \& Elmegreen (1998). Below, we report and describe such systems observed in IC 2574.

\subsection{Groups Related to the SGS}

The SGS is an impressive Hi expanding shell located in the northwest region of IC 2574 and created by a significant burst of star formation in its center. It is likely responsible for triggering a ring of star formation around it (Walter et al. 1998; Walter \& Brinks 1999; Pasquali et al. 2008). Many groups have been found with HOP all around the SGS, as well as within, including fairly dispersed groups. This is the case for $01 \mathrm{~b}, 01 \mathrm{~d}$, 02b, 07c, 08a, and 28b. Groups that were found within the SGS are easily identified with HOP and have numbers between $01 \mathrm{z}$ and $24 z$, all having the " $z$ " extension. They include some of the densest groups found by HOP in the images. Among the groups around the SGS, half belong to category I while the other half to category III. The highly dispersed groups from category III seem to be part of a more general diffuse field of blue stars (20-40 Myr). In fact, most stellar groups within and around the SGS are superimposed to a generous diffuse stellar background of blue stars and evolved bright red supergiants consistent with an age of $\sim 20-40$ Myr. Such diffuse structure was observed and characterized in detail by Maíz-Apellániz (2001), but is not clear in this case if this diffuse stellar population is a remnant of the starburst that created the SGS.

\subsection{Groups Related to the Giant H II Region}

The ACS field of view includes a giant H II region, located on the opposite end (roughly south) of the SGS in Figure 3. The whole region with the strong nebular emission is about $250 \mathrm{pc}$ in projected size. Within the region, HOP easily identified five stellar groups, namely 50z, 51z, 52z, 53z, and 54z. Like the SGS, it is surrounded by a sparse population of bright blue stars and red supergiants. Around the giant $\mathrm{H}$ II region, HOP identified several fairly sparse groups, namely, 74c, 75e, 76d, $77 \mathrm{a}, 80 \mathrm{c}$, and 81c. Interestingly, except for the young groups $74 \mathrm{c}$ and 75 e displaying nebular emission, all the other groups show evolved red stars consistent with an age of around $40 \mathrm{Myr}$. This suggests that there is an underlying stellar generation born a few $10^{7} \mathrm{yr}$ before the current young $\mathrm{H}$ II region content. The stellar population cannot be much younger since we clearly detect evolved red supergiants. It is unclear if the older generation produced a more intense and/or more voluminous star formation burst than the current one.

\subsection{Other Small Complexes}

Some other groups could be physically linked to each other based on their proximity. This is the case for $11 \mathrm{a}+11 \mathrm{c}+13 \mathrm{a}$, $25 \mathrm{c}+25 \mathrm{e}+25 \mathrm{~g}+25 \mathrm{~h}+25 \mathrm{i}, 18 \mathrm{c}$ which contains several subgroups, $54 c+55 b+56 a, 71 a+71 b+71 c$, and $30 b+30 d$. Two groups, $15 a$ and $30 \mathrm{~d}$, display a very elongated projected shape. They may have been the result of star formation along a filament (Gutermuth et al. 2008). It is interesting to note that groups within these complexes can have a spread of ages. All these groups are good candidates for systems formed in a hierarchical manner through the fragmentation of their parent molecular cloud.

\section{DISPERSING COMPACT GROUPS}

In this work, we are also interested in looking for compact stellar groups that are undergoing rapid dissolution. With HOP, we found very few compact and young groups for which this could be the case, namely, 03a, 25h, 56a, and 83d. We also find a few within the SGS and the giant $\mathrm{H}$ II region that have a very compact core and could be partly dispersing (e.g., 01z, 04z, 05z, $22 \mathrm{z}, 24 \mathrm{z})$. It is somewhat surprising that we do not find more of this kind of group in IC 2574. In a scenario where 50\%-90\% of the star clusters/groups disrupt and dissolve within the first $10^{7} \mathrm{yr}$, and an additional $50 \%-90 \%$ between $10^{7}$ and $10^{8} \mathrm{yr}$ (e.g., the Antennae; Fall et al. 2005), it would be reasonable to expect more than a handful of compact dissolving candidates given the recent star formation history of IC 2574. However, Baumgardt \& Kroupa (2007), Fall et al. (2009), and Portegies Zwart et al. (2010) suggested that the dispersal time for an unbound cluster is at most $10 \mathrm{Myr}$ or $\sim 10$ crossing times. According to these works, it would mean that most star formation occurs in unbound clusters/groups and would explain why so few compact young dissolving systems are observed in IC 2574.

\section{CORRELATION BETWEEN H I GAS AND HOP STELLAR GROUPS}

Walter \& Brinks (1999) have studied the H I gas in IC 2574 in great detail. They cataloged 48 holes, which they argue are most likely the result of stellar winds and supernova explosions of young stellar systems, such as young star clusters and OB associations. They conclude that the lack of shear forces in dwarf galaxies like IC 2574 contribute to the longer-term survival of the numerous $\mathrm{Hi}$ holes they found as well as their spherical shapes. Pasquali et al. (2008) investigated the presence of young stellar populations within those $\mathrm{H}_{\mathrm{I}}$ holes using pixel-by-pixel maps from ground-based data and could not find any clear association between the two.

At this point, it is interesting to check if the loose stellar groups we find with HOP could be the cause of at least some of these H I holes. Low surface brightness dispersed groups could have been missed in the $U$-band image used by Pasquali et al. (2008). The presence of both an $\mathrm{H}$ I hole and a loose stellar group would be consistent with stellar winds and supernova being involved in the infant mortality of star clusters.

We compared the location of all the stellar groups reported in Tables 2-4 together with the list of $\mathrm{H}_{\mathrm{I}}$ holes cataloged by Walter \& Brinks (1999). The holes found have dynamical ages $\sim 10-60$ Myr which correspond well to the age range of the HOP groups. Unfortunately, we do not find any correlation at all 
within the HST/ACS field of view covered in our work, except for the SGS as already studied by Walter et al. (1998).

Since we find that there are neither compact star clusters nor dispersed groups associated with the H i holes in IC 2574, this leads to the possibility that Type Ia supernovae could be at the origin of these interstellar medium (ISM) features. That would also be in agreement with the typical energy released to create the hole and calculated by Walter \& Brinks (1999). The presence of SN Ia do not require a young stellar population, as for stellar winds and core-collapse SN, and they can be located anywhere within the galaxy. We calculated the SN Ia rate in IC 2574 based on the study from Li et al. (2011), assuming a stellar mass of $\sim 5 \times 10^{8} M_{\odot}$ for IC 2574 . In the past $50 \mathrm{Myr}$, which is the age range measured for the $\mathrm{H}$ i holes, IC 2574 should have seen a few $10^{3} \mathrm{SN}$ Ia. This is an order of magnitude larger than the number of holes cataloged by Walter \& Brinks (1999). However, as mentioned by the authors, the $\mathrm{H}$ i hole catalog is incomplete considering that ISM features smaller than $100 \mathrm{pc}$ are excluded due to their spatial resolution. Although the uncertainties are large, an SN Ia is a more plausible cause of the Hi holes in IC 2574 than recent star formation.

Finally, we compared the location of the HOP groups with the location of H I gas, as presented by Walter \& Brinks (1999, their Figure 11). We find a strong correlation between the two, with HOP groups aligned with regions of higher $\mathrm{H}_{\mathrm{I}}$ surface brightness, with a few exceptions. This correlation strengthens the case for our resolved star approach finding real systems and not asterisms.

\section{CONCLUSION}

In this work, we have developed and explored a new approach to search for dispersed groups of stars that could be dissolving stellar systems that are very difficult to detect from traditional photometric techniques. The approach is based on photometry of individual stars in nearby galaxies. Using a coordinate list of bright blue stars as input, we used the elaborated friendsof-friends algorithm HOP (Eisenstein \& Hut 1998) to retrieve overdensities of stars. We characterized those groups using color-magnitude and stellar density radial profiles to estimate their age, size, densities, and shapes.

As a test case, we used a field of IC 2574 observed with ACS on board HST and found 75 dispersed stellar groups, including those within a large SGS and an H II region. We separated these groups into three categories: (I) groups with nebular emission, (II) groups without nebular emission, and (III) highly dispersed groups. Using this new method on IC 2574, we found:

1. twenty highly dispersed groups, which may or may not be asterisms;

2. only four compact dissolving candidates. Assuming a scenario where $50 \%-90 \%$ of clusters and groups dissolve within the first $10^{7} \mathrm{yr}$, we would expect to find more of such candidates given the recent star formation history of IC 2574. Based on the theoretical work of Fall et al. (2005) and Baumgardt \& Kroupa (2007), where they found a dispersal time for an unbound cluster of $\sim 10^{7} \mathrm{yr}$, it would mean that most star formation in IC 2574 occurred in unbound clusters/groups;

3 . that the median group dispersion speeds are at most $3.9 \pm$ 2.7, $1 \pm 1$, and $1 \pm 1 \mathrm{~km} \mathrm{~s}^{-1}$ for groups in categories I, II, and III, respectively;

4. that the most massive stars are bad tracers of size and shape of loose groups;
5. that the shape and orientation of the groups do not show a pattern that would indicate an external and organized force, such as shears or density waves, acting to disrupt and dissolve the groups, which is consistent with a solid body rotation for IC 2574;

6. that there is a trend for older groups to be larger, suggesting a dispersion rate of $0.45 \mathrm{pc} \mathrm{Myr}^{-1}$, assuming that all groups found in this work are not asterisms;

7. that several groups are located near each other and may be physically related to a common parent cloud;

8. that the location of HOP groups correlates well with the $\mathrm{H}_{\mathrm{I}}$ contour map features;

9. that the HOP groups do not correlate with the H I holes found by Walter \& Brinks (1999), suggesting another origin for the holes such as SN Ia.

Overall, this method is good at finding non-compact groups of stars. However, it is a laborious process because it requires repetitive visual inspections at various stages, making it very difficult to automate.

We acknowledge financial support from HST-AR-10968 and HST-AR-12148 grants. This research has made extensive use of NASAs Astrophysics Data System and of the NASA/IPAC Extragalactic Database (NED) which is operated by the Jet Propulsion Laboratory, California Institute of Technology, under contract with the National Aeronautics and Space Administration.

\section{NOTES ON INDIVIDUAL STELLAR GROUPS}

$01 b$. This group can be detected by eye and is an excellent candidate for a coeval stellar population. The CMD and isochrones indicate the presence of main-sequence stars only with an age of $15 \mathrm{Myr}$ or younger. Prominent nebular emission suggests an upper age limit of 10 Myr. It is located around the SGS.

01d. This is a dispersed group around the SGS. The CMD is consistent with a population of $\leqslant 40 \mathrm{Myr}$. It may well be a remnant of the star-forming episode that created the SGS. The group size is difficult to estimate since the density profile does not flatten.

$01 z$. This group is located within the SGS and is easily found by HOP. It contrasts well with the surrounding diffuse field on the density profile. The CMD indicates a very young cluster of 10-15 Myr. This is consistent with the residual nebular emission located in a shell around the group. The group is also characterized with a denser core superimposed onto a more dispersed population of stars, as described by Maíz-Apellániz (2001). The core is contaminated by a foreground star.

$02 b$. This is a good case of a very dispersed group that does not stand out well from the diffuse field on the CMD, but still shows a clear overdensity on the density profile. Its coeval property is disputable. The group is in the peripheral region of SGS and cannot be much younger than $40 \mathrm{Myr}$.

$02 z$. This is a large and fairly dispersed young group with faint nebular emission. The CMD suggests a dual population, one with several evolved red stars of 30-40 Myr plus another younger one of $\sim 10$ Myr. The size of this dispersed group is not clear, since the background level is quite high in the SGS region.

03a. This small group has a fairly compact core, although it contains few stars. They are very consistent with a population of $\sim 40 \mathrm{Myr}$, with two evolved red stars on the isochrone. 
03z. This is a fairly dispersed group within the SGS which shows faint nebular emission that may or may not be associated with it. It does not stand out particularly well from its surroundings according to the density profile. The CMD suggests a dual population, one around $40 \mathrm{Myr}$ and a younger one on the 10-16 Myr isochrone.

04z. This young group is at the center of the SGS and is possibly responsible for the $\mathrm{H}$ I shell. No nebular emission is associated with this group. The CMD shows a few objects on evolutionary tracks younger than $40 \mathrm{Myr}$, but they are likely stellar blends or multiple unresolved stars since there is a highly dense core at the center of the group.

$05 a$. This group contains a small number of stars, but one is very bright and two are surrounded by faint nebular emission, which helps to constrain the age to no more than 10-15 Myr. The density profile is fairly sharp and indicates a group size of $\sim 40 \mathrm{pc}$.

05z. This is a very interesting group within the SGS with a very bright and compact core. The density profile shows a hole at the center since no individual star can be resolved. The CMD indicates an age around 15-20 Myr, if we ignore the brighter sources that are most likely stellar blends. It is interesting to note the high background level in this region of the galaxy.

06z. This is probably a random grouping within the local diffuse field of the SGS. There is nothing obvious on the density profile. If this is typical of the local diffuse field, then it indicates a stellar population of 30-40 Myr.

07c. This group surrounds a young and bright $\mathrm{H}$ II region triggered by the SGS. The CMD shows a stellar group of about 15 Myr. However, it may be younger because of the nebular emission seen with this group, although the gas may also be ionized by the nearby $\mathrm{H}$ II region. The density profile shows an irregular profile, with an overdensity at the center and a lack of stars at a radius of $\sim 30 \mathrm{pc}$ compared to the general background density. It may be a combined effect of the general irregular shape of the loose stellar group and the high diffuse field level.

08a. This group is very sparse and barely stands out on the density profile. It may well be part of the local diffuse field related to the SGS generation or simply an asterism. It is at most 20-40 Myr.

11a. This is another sparse group, as shown by the density profile. One bright blue star on the CMD suggests an age of $\sim 30$ Myr or younger. Nebular emission is seen around one star only.

11c. This group, next to $11 \mathrm{a}$, contains a very small number of stars and shows a faint and extended nebular emission. This group contains a few blue stars clustered together seen with HOP, but this does not show up in the density profile. The CMD suggests an age of at most $40 \mathrm{Myr}$, but the faint nebular emission associated with it indicates an age younger than $10 \mathrm{Myr}$.

13a. This is a very young group with nebular emission easily picked up by HOP. This group stands out easily from the diffuse field. Its density profile would be consistent with an $\mathrm{OB}$ association or a scaled OB association (Maíz-Apellániz et al. 2004), depending on its mass. Its shape and size are difficult to establish since the density profile does not flatten.

13z. This is probably the brightest group associated with the SGS. It is very large (90 pc) and elongated. It displays strong nebular emission clearly related to a $<10$ Myr population, which is also consistent with the CMD. The core is very crowded and contains at least five highly compact sub-groups. The density profile confirms the size and shape of the imposing group.
$15 a$. This group is very sparse. It is located near the detector edge and may be part of a bigger system. A few red stars on the CMD suggests an age around $40 \mathrm{Myr}$. A bright yellow star appears on the instability strip of the CMD and may well be a Cepheid star ( $\alpha=10: 29: 00.77, \delta=+68: 28: 29.0$ in J2000). The density profile shows a hole in the center of the group, which may indicate that the group formed along filaments within the molecular cloud.

15z. This is a very young and bright group for which most stars cannot be resolved. It has a strong nebular emission, and is therefore younger than $10 \mathrm{Myr}$. Four yellow stars on the CMD are good Cepheid candidates (in J2000: $\alpha_{1}=10: 28: 47.9$, $\delta_{1}=+68: 28: 04.9 ; \alpha_{2}=10: 28: 48.4, \delta_{2}=+68: 28: 05.0$; $\alpha_{3}=10: 28: 47.9, \delta_{3}=+68: 28: 05.4 ; \alpha_{4}=10: 28: 48.5, \delta_{4}=$ $+68: 28: 01.8$ ).

16c. This is an isolated group with a CMD generally consistent with an age of 40 Myr. One star may be a Cepheid on the instability strip ( $\alpha=10: 28: 55.5, \delta=+68: 27: 57.9$ in J2000). One very bright blue star is not consistent with a $40 \mathrm{Myr}$ age, and may be a stellar blend.

18c. This group is close to an H II region and contains several smaller subgroups. This picture would fit well within the hierarchical star formation theory (i.e., Efremov 1995; Efremov \& Elmegreen 1998; Elmegreen et al. 2000; Elmegreen 2002). The group is dispersed but quite rich in its number of bright blue stars. The CMD is consistent with an age of 20-40 Myr, although each subgroup could have slightly different ages, as suggested by a small group of stars with clear nebular emission. Several background galaxies are seen in this region of IC 2574. A bright yellow star located on the instability strip of the CMD is a good Cepheid candidate $(\alpha=10: 28: 53.7, \delta=+68: 28: 35.5$ in $\mathrm{J} 2000$ ).

18z. This is a small group in the SGS of a few bright stars. Some sources may be the result of blends. According to the CMD, this group is at most $20 \mathrm{Myr}$.

19z. This is a fairly small and dispersed group in the SGS without nebular emission. The CMD indicates a population younger than $16 \mathrm{Myr}$. The density profile is quite noisy.

$20 z$. This is a dispersed group in the SGS without nebular emission. The CMD indicates an age around 10-15 Myr, together with a few evolved red stars on the $\sim 40 \mathrm{Myr}$ isochrone. This group does not stand out much from the high diffuse field level. It may be an asterism.

21c. This is an extremely dispersed group which is basically part of the diffuse field population, possibly an asterism, with an age of $100 \mathrm{Myr}$.

$21 z$. This is a bright star-forming group within the SGS with strong nebular emission. The nebular emission is consistent with a CMD age of $10 \mathrm{Myr}$, at most. This group contains several subgroups, some of which display a very high stellar density with unresolved stars.

$22 z$. This is a bright and young stellar group in the SGS and probably linked to group $21 \mathrm{z}$. The strong nebular emission and the CMD suggest an age younger than $10 \mathrm{Myr}$. A bright red star is observed in this group and is probably part of the underlying diffuse field population. The noisy density profile suggests a size between 15 and $30 \mathrm{pc}$.

$23 c$. This is a fairly isolated, young, and compact group, with a low stellar background level. It displays strong nebular emission around a few stars, which indicate an age under 10 Myr.

23z. This relatively small group $(20-30$ pc) stands out relatively well from the density profile. It produces a faint 
and diffuse nebular emission, in agreement with the CMD age younger than 15 Myr.

$24 z$. This group shows a very bright and compact component asymmetrically overlaid on a more diffuse distribution of stars. Due to the asymmetry, it is difficult to precisely establish the size of the group because it interferes with the density profile. As a result of the strong nebular emission, the CMD displays multiple stars bluer than the main sequence. None of the brightest stars are red and evolved, consistent with the young age of $<10 \mathrm{Myr}$ already suggested by the nebular emission.

$25 c$. This is a fairly compact and young group with bright nebular emission, setting its age under $10 \mathrm{Myr}$ which is consistent with the CMD. This is part of a larger group that includes several substructures. A visual inspection indicates signs that the central part may contain a dense core with unresolved stars. The CMD shows two yellow stars that are good candidates for being Cepheid variables $\left(\alpha_{1}=10: 28: 37.40, \delta_{1}=\right.$ $\left.+68: 27: 58.3 ; \alpha_{2}=10: 28: 37.45, \delta_{2}=+68: 27: 57.6\right)$.

$25 e$. This young and small group with nebular emission is part of the larger group with $25 \mathrm{c}$. It shows elongated features that may be unresolved stars. The density profile suggests a size between 10 and $40 \mathrm{pc}$.

$25 \mathrm{~g}$. This group contains very few stars, as can be seen in the CMD and by the noise on the density profile. It is part of the larger group together with $25 \mathrm{c}$ and $25 \mathrm{e}$. It displays some nebular emission, in agreement with the CMD age estimation.

$25 \mathrm{~h}$. This is at the center of the larger group that includes $25 \mathrm{c}, 25 \mathrm{e}$, and $25 \mathrm{~g}$. Some nebular emission is detected in the $\mathrm{H} \alpha$ image at the location of this group, but it is not noticeable in the broadband images. This provides an age not much older than 10-15 Myr for this group, consistent with the CMD.

25i. This small HOP group is part of the larger group with $25 \mathrm{cegh}$, but is much more sparse and does not show signs of nebular emission. It barely stands out of the diffuse field population, as can seen on the image and the density profile. The CMD strongly suggests an age around 40 Myr.

$28 b$. This is a very sparse group with faint nebular emission, located at the edge of the SGS. The presence of nebular emission is consistent with the few bright blue stars on the main sequence of the $<16$ Myr Padova isochrones.

29f. This is a very small group around an $\mathrm{H}$ II region. This is actually an OB group next to a more intense star-forming system. There is strong nebular emission associated with one or a few stars.

$30 b$. This group is fairly isolated and sparse, and yet still pretty young with two bright stars, one blue and one red, that allow us to constrain the age to 10-40 Myr at most. A very faint nebular emission can be see on the F555W image. The center of the group and its size are very difficult to estimate since the system is very asymmetric.

30d. This is an interesting group because it is fairly isolated, not particularly young $(\sim 100 \mathrm{Myr})$, and yet it stands out from the diffuse field population in the CMD and in the density profile. It is an excellent candidate for a dissolving system.

$34 b$. This is a group with a very few number of bright blue stars, but it stands out from the diffuse field population on the density profile. Its age is difficult to estimate, with only one star brighter than F555W $=24$ mag on the $40 \mathrm{Myr}$ isochrone. This bright central star shows clear emission in the $\mathrm{H} \alpha$ and may also be a stellar blend.

$38 c$. This is a nice sparse group with a low number of stars for which its age is between 10 and 40 Myr. However, the brightest star is possibly a contaminating foreground star, which would bring the age to about $40 \mathrm{Myr}$.

$40 b$. This group was easily picked up by HOP, but curiously the density profile does not show in it anything more compact than the surrounding diffuse field. It is a very young group with no nebular emission, and not compact at all. The group is clearly distinct from the older diffuse field population on the CMD, and its age is $\leqslant 15$ Myr.

4la. This group stands out with HOP because of the concentration of bright blue stars, but it is as sparse as the local diffuse field density according to the density profile. It is quite young, probably at most $15 \mathrm{Myr}$, but a few yellow stars falling on the $40 \mathrm{Myr}$ isochrone give cause for doubt. One of those yellow stars is associated with compact nebular emission that is better seen in the $\mathrm{H} \alpha$ image.

47b. This is a very sparse group identified by HOP, with a density profile suggesting an overdensity. The CMD shows a few bright blue stars consistent with an age of $20 \mathrm{Myr}$ or younger. It may well be an asterism.

$49 a$. This group is probably part of the diffuse field population. The density profile is quite flat but it also has a quite high diffuse field density. The CMD shows many stars on the $100 \mathrm{Myr}$ isochrone that are fairly well separated from the field stars. It is a good candidate for a highly dissolved system. Its size and shape are not possible to establish.

$49 c$. This is a very small group with faint nebular emission. It contains a few stars that are consistent with a $\sim 20$ Myr isochrone or younger.

50z. This group belongs to the giant $\mathrm{H}$ II region and is associated with strong nebular emission. This emission is in agreement with the CMD age of $<10 \mathrm{Myr}$.

51a. This is an isolated and dispersed group that includes one bright blue star, consistent with a 15 Myr isochrone or younger. Without that one star, an age closer to $100 \mathrm{Myr}$ would be appropriate given the CMD. The group barely stands out from the diffuse stellar field population. It may well be an asterism.

$51 z$. This group within the giant $\mathrm{H}$ II region shows some nebular emission in its outskirts. This is consistent with an age of 10-15 Myr suggested by the CMD fitting.

$52 z$. This small group ( $\sim 20 \mathrm{pc})$ displays strong nebular emission and contributes to the brightness of the giant $\mathrm{H}_{\text {II }}$ region. This age is consistent with the CMD fitting of $<40 \mathrm{Myr}$ and is based on a fairly limited amount of stars.

53z. This group is fairly compact and does not show obvious nebular emission directly associated with it. The CMD and isochrone fitting indicate an age of 10-15 Myr, although the few bright blue stars may be stellar blends. It is easily picked up with HOP but is not clear on the density profile.

$54 c$. This is a very isolated and small group that stands out very well from the diffuse field population on the density profile. It was easy to identify it with HOP. According to the CMD, it is probably about 40-60 Myr. Two of the brightest stars are located on the instability strip and may be Cepheids, depending on their reddening (in J2000: $\alpha_{1}=10: 29: 00.58, \delta_{1}=+68: 26: 43.13$; $\alpha_{2}=10: 29: 00.81, \delta_{2}=+68: 26: 42.9$ ).

$54 z$. This is a dispersed group within the giant $\mathrm{H}$ II region. Faint, diffuse nebular emission can be detected, which is consistent with a CMD fitting of $<15 \mathrm{Myr}$. The $\sim 40$ pc system is well seen on the density profile.

$55 b$. This is an isolated and small dispersed group. The density profile shows a clear detection. However, the age is somewhat difficult to estimate based on the CMD due to the very low number of bright stars in the group and the fact that there is 
no bright yellow or red star to constrain the isochrones. The brightest blue star, if it is not a multiple system, suggests an age of $20 \mathrm{Myr}$ or younger. If we ignore the brightest blue star, then we obtain an age of $\leqslant 50 \mathrm{Myr}$.

$56 a$. This group is located at the very edge of the detector, and may not be complete. The CMD suggests and age younger than $15 \mathrm{Myr}$, consistent with the nebular emission observed in the F555W filter and the $\mathrm{H} \alpha$ image.

$57 c$. This is a sparse group clearly detected by HOP, but it does not stand out on either the density profile or the CMD. The CMD shows a few stars on the main sequence that are consistent with a $\leqslant 30$ Myr isochrone, but two very red stars may extend the age up to $\sim 70 \mathrm{Myr}$, depending on the reddening. This group is a good candidate for a highly dissolved coeval system.

$58 a$. This group is very small. It is located right next to a compact star cluster with unresolved stars. The group does not show signs of nebular emission and two stars on the CMD suggest an age estimation of $\leqslant 20 \mathrm{Myr}$. The group does not stand out much on the density profile.

60e. This small group is fairly isolated and dispersed, but does not have bright blue stars standing out from the older diffuse field in the CMD. The density profile shows a small overdensity from the diffuse field. The age of its brightest stars would be between 40 and $100 \mathrm{Myr}$, according to the isochrones.

$61 c$. This is a very small group with a few stars that could fit $\mathrm{a} \leqslant 40 \mathrm{Myr}$ system. The density profile is very noisy.

$62 c$. This group is very sparse and would not have been seen without HOP. It is located near the detector edge. The CMD suggests a population of $\sim 40$ Myr. The density profile is unreliable at $r>120$ pc because it falls outside of the detector. This is a good candidate for a dissolving coeval system.

$67 d$. This is a sparse group with few stars, and is close to the group 40b. The CMD suggests an age of $40 \mathrm{Myr}$, although it is contaminated by stars of older generations. The density profile shows an overdensity at this location within a $40 \mathrm{pc}$ radius.

$69 e$. This group clearly stands out from the diffuse field population, even though the density profile is biased by the edge of the detector for $r>60 \mathrm{pc}$. The CMD fits an age of $20 \mathrm{Myr}$ or younger. No nebular emission is detected. The group is contaminated by background galaxies. One bright yellow star may be a Cepheid $(\alpha=10: 28: 38.62, \delta=+68: 25: 41.1$ in $\mathrm{J} 2000$ ).

70b. This is an extremely dispersed group with significant contamination. It is a good candidate for a dissolving cluster. It stands out marginally from the density profile. Its size and shape are very difficult to establish. The star at $\alpha=10: 28: 41.28 \delta=$ $+68: 25: 52.4$ may be a Cepheid.

$71 a$. This is a nice loose group. It is overdense on the density profile and there are several yellow and red stars on the $40 \mathrm{Myr}$ isochrone. One blue star shows very faint nebular emission. Another is a good Cepheid candidate given its CMD location on the instability strip $(\alpha=10: 28: 44.73, \delta=+68: 25: 44.17$ in J2000).

$71 b$. This group, located next to $71 \mathrm{a}$, is very sparse. It is about $30 \mathrm{Myr}$, according to the CMD and isochrones. The stellar overdensity is relatively weak.

71c. This dispersed group is located near 71a and 71b. There are many bright blue stars on the CMD but they do not clearly converge toward a consistent age. It could be around $50 \mathrm{Myr}$ if we ignore the brightest star or $\leqslant 15 \mathrm{Myr}$ if we assume that a few yellow stars are in fact main-sequence stars affected by some level of reddening. There is a faint detection of nebular emission in the F555W filter as well as in the $\mathrm{H} \alpha$ image, which strengthens the younger age hypothesis. The density profile suggests a weak overdensity in this location.

71e. This group is a good candidate for a dissolving system. It is contaminated by a background galaxy. Several stars lying on the $\sim 40-60$ Myr isochrone separate fairly well from the diffuse field population. Two stars may be on the instability strip, depending on the reddening $\left(\alpha_{1}=10: 28: 43.94, \delta_{1}=\right.$ $+68: 25: 52.5 ; \alpha_{2}=10: 28: 44.67, \delta_{2}=+68: 25: 51.8$ in J2000)

$72 b$. This is a nice young group, rich in bright blue stars with nebular emission seen in $\mathrm{H} \alpha$, with an age or $10 \mathrm{Myr}$ at most, which is supported by the CMD. The density profile shows a clear overdensity that stands out from the diffuse field population.

$74 c$. This is a nice group, fairly compact, and showing clear nebular emission, which makes it younger than 10 Myr. The density profile shows a clear stellar overdensity. The group may be linked to the giant $\mathrm{H}$ II region.

$75 e$. This is a nice sparse group that stands out clearly on the stellar density profile. It is associated with some nebular emission inferring an age younger than $10 \mathrm{Myr}$, as supported by the CMD. It is probably linked to the giant $\mathrm{H}$ II region.

$76 \mathrm{~d}$. This group stands out from the density profile as well as on the CMD. It is about $40 \mathrm{Myr}$, but may be mixed with a 100 Myr population, depending on the reddening of two yellow stars. It is located next to a compact star cluster and is probably linked to the giant $\mathrm{H}$ II region.

$77 a$. This group is fairly dispersed and shows several stars consistent with a $\sim 40 \mathrm{Myr}$ isochrone. However, the group is located in the outskirts of the giant H II region and the density profile is not clear about an overdensity. The group is probably part of the formation process related to the Hil region.

$80 c$. This group is sparse and located in the outskirts of the large $\mathrm{H}$ II region. The group stands out both in the CMD and the density profile. A few evolved bright stars are consistent with the 30-40 Myr isochrone. No nebular emission is seen with the group.

$81 c$. This group is located at the very edge of the detector. The density profile is not fully reliable because of that. The CMD however shows a stellar population of $40 \mathrm{Myr}$ or younger. No nebular emission is seen.

$83 \mathrm{~d}$. This group is made up of a good number of bright blue stars and looks pretty compact on the F435W image. Interestingly, the density profile does not show a clear overdensity. The group is associated with diffuse nebular emission and may be part of a bigger group. The young stars stand out very nicely on the CMD, and the age is estimated to $<10 \mathrm{Myr}$.

\section{REFERENCES}

Anders, P., Bissantz, N., Fritze-v. Alvensleben, U., \& de Grijs, R. 2004, MNRAS, 347,196

Bastian, N. 2011, in Stellar Clusters \& Associations: A RIA Workshop on Gaia, Cluster Disruption: From Infant Mortality to Long Term Survival, ed. E. J. A Navarro \& A. T. G. Calvente, 85

Bastian, N., Adamo, A., Gieles, M., et al. 2012, MNRAS, 419, 2606

Bastian, N., \& Goodwin, S. P. 2006, MNRAS, 369, L9

Bastian, N., Trancho, G., Konstantopoulos, I. S., \& Miller, B. W. 2009, ApJ, 701, 607

Bastian, N., Weisz, D. R., Skillman, E. D., et al. 2011, MNRAS, 412, 1539

Bate, M. R. 2009, MNRAS, 392, 590

Baumgardt, H., \& Kroupa, P. 2007, MNRAS, 380, 1589

Baumgardt, H., \& Makino, J. 2003, MNRAS, 340, 227

Bonnell, I. A., \& Bate, M. R. 2006, MNRAS, 370, 488

Cannon, J. M., Walter, F., Bendo, G. J., et al. 2005, ApJ, 630, L37

Chandar, R., Fall, S. M., \& Whitmore, B. C. 2006, ApJ, 650, L111

Chandar, R., Fall, S. M., \& Whitmore, B. C. 2010, ApJ, 711, 1263 
Chandar, R., Whitmore, B. C., Calzetti, D., et al. 2011, ApJ, 727, 88

Croxall, K. V., van Zee, L., Lee, H., et al. 2009, ApJ, 705, 723

Dalcanton, J. J., Williams, B. F., Seth, A. C., et al. 2009, ApJS, 183, 67

Dale, J. E., Bonnell, I. A., Clarke, C. J., \& Bate, M. R. 2005, MNRAS, 358,291

Efremov, Y. N. 1995, AJ, 110, 2757

Efremov, Y. N., \& Elmegreen, B. G. 1998, MNRAS, 299, 588

Eisenstein, D. J., \& Hut, P. 1998, ApJ, 498, 137

Elmegreen, B. G. 2002, ApJ, 564, 773

Elmegreen, B. G. 2011, in EAS Publications Series, Vol. 51, Star Formation Patterns and Hierarchies, ed. C. Charbonnel \& T. Montmerle (Cambridge: Cambridge University Press), 31

Elmegreen, B. G., Efremov, Y., Pudritz, R. E., \& Zinnecker, H. 2000, in Protostars and Planets IV, Observations and Theory of Star Cluster Formation, ed. V. Mannings, A. P. Boss, \& S. S. Russell (Tucson, AZ: Univ. Arizona Press), 179

Elmegreen, B. G., \& Hunter, D. A. 2010, ApJ, 712, 604

Fall, S. M., Chandar, R., \& Whitmore, B. C. 2005, ApJ, 631, L133

Fall, S. M., Chandar, R., \& Whitmore, B. C. 2009, ApJ, 704, 453

Fall, S. M., \& Zhang, Q. 2001, ApJ, 561, 751

Fellhauer, M., \& Kroupa, P. 2005, ApJ, 630, 879

Georgiev, T. B., Tikhonov, N. A., Karachentsev, I. D., \& Ivanov, V. D. 1996, Astron. Astrophys. Trans., 11, 39

Gieles, M., Bastian, N., Lamers, H. J. G. L. M., \& Mout, J. N. 2005, A\&A, 441, 949

Gieles, M., Portegies Zwart, S. F., Baumgardt, H., et al. 2006, MNRAS, 371,793
Girichidis, P., Federrath, C., Banerjee, R., \& Klessen, R. S. 2011, MNRAS, 413, 2741

Goodwin, S. P., \& Bastian, N. 2006, MNRAS, 373, 752

Gutermuth, R. A., Myers, P. C., Megeath, S. T., et al. 2008, ApJ, 674, 336

Karl, S. J., Fall, S. M., \& Naab, T. 2011, ApJ, 734, 11

Kruijssen, J. M. D., Inti Pelupessy, F., Lamers, H. J. G. L. M., et al. 2011, MNRAS, 421, 1927

Kruijssen, J. M. D., Maschberger, T., Moeckel, N., et al. 2012, MNRAS, 419,841

Lada, C. J., \& Lada, E. A. 2003, ARA\&A, 41, 57

Lamers, H. J. G. L. M., Gieles, M., Bastian, N., et al. 2005, A\&A, 441, 117

Larsen, S. S. 2004, A\&A, 416, 537

Li, W., Chornock, R., Leaman, J., et al. 2011, MNRAS, 412, 1473

Maíz-Apellániz, J. 2001, ApJ, 563, 151

Maíz-Apellániz, J., Pérez, E., \& Mas-Hesse, J. M. 2004, AJ, 128, 1196

Pasquali, A., Leroy, A., Rix, H.-W., et al. 2008, ApJ, 687, 1004

Portegies Zwart, S. F., McMillan, S. L. W., \& Gieles, M. 2010, ARA\&A, 48, 431

Schlegel, D. J., Finkbeiner, D. P., \& Davis, M. 1998, ApJ, 500, 525

Sirianni, M., Jee, M. J., Benítez, N., et al. 2005, PASP, 117, 1049

Tremonti, C. A., Calzetti, D., Leitherer, C., \& Heckman, T. M. 2001, ApJ, 555,322

Walter, F., \& Brinks, E. 1999, AJ, 118, 273

Walter, F., Kerp, J., Duric, N., Brinks, E., \& Klein, U. 1998, ApJ, 502, L143

Weisz, D. R., Skillman, E. D., Cannon, J. M., et al. 2008, ApJ, 689, 160

Weisz, D. R., Skillman, E. D., Cannon, J. M., et al. 2009, ApJ, 691, L59

Whitmore, B. C., Zhang, Q., Leitherer, C., et al. 1999, AJ, 118, 1551 